\title{
Construction identitaire de l'élève en éducation artistique dans un contexte francophone minoritaire canadien
}

\section{Mariette Théberge}

\section{CpenEdition}

Journals

Édition électronique

URL : http://journals.openedition.org/trema/2546

DOI : $10.4000 /$ trema.2546

ISSN : 2107-0997

Éditeur

Faculté d'Éducation de l'université de Montpellier

Édition imprimée

Date de publication : 1 décembre 2010

Pagination : 64 - 71

ISSN : 1167-315X

Référence électronique

Mariette Théberge, «Construction identitaire de l'élève en éducation artistique dans un contexte francophone minoritaire canadien », Tréma [En ligne], 33 - 34 | 2010, mis en ligne le 01 décembre 2012, consulté le 10 décembre 2020. URL : http://journals.openedition.org/trema/2546 ; DOI : https:// doi.org/10.4000/trema.2546

Ce document a été généré automatiquement le 10 décembre 2020.

Trema 


\title{
Construction identitaire de l'élève en éducation artistique dans un contexte francophone minoritaire canadien
}

\author{
Mariette Théberge
}

1 Dans les cinq dernières années, plusieurs approches d'éducation artistique et politiques linguistiques ont été mises en œuvre dans le contexte de la francophonie canadienne pour favoriser la construction identitaire de l'élève (Association canadienne d'éducation de langue française, 2006; Fédération culturelle canadienne française, 2009). Par exemple, participant à une prise de conscience du

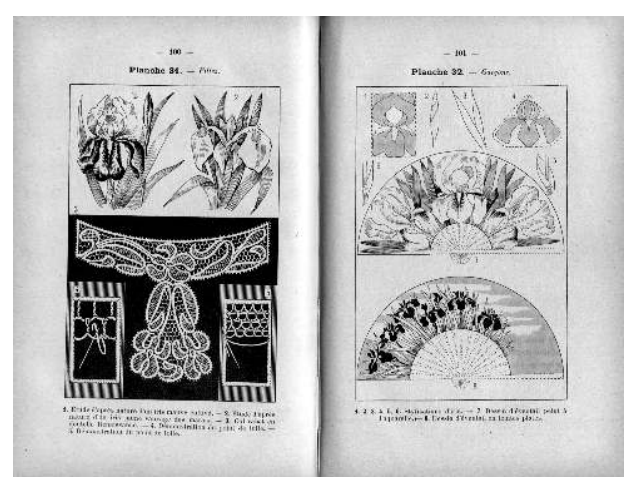
rôle de l'école dans la société, le ministère de l'Éducation de l'Ontario s'est doté en 2004 d'une Politique d'aménagement linguistique qui reconnaît des liens explicites entre les manifestations artistiques et culturelles vécues à l'école et le sens d'appartenance à la communauté francophone. De plus, ce même ministère encourage l'instauration de programmes spécialisés en arts pour les élèves de 14 à 18 ans et a mis sur pied en 2006 le programme Majeure Haute Spécialisation en Arts et Culture; ce programme permet pour ces élèves de consolider la place de la formation artistique dans le cadre scolaire ontarien et de favoriser une concertation avec les contextes culturels et artistiques professionnels (ministère de l'Éducation de l'Ontario, 2004 ; 2006). Pour montrer un tant soit peu des démarches qui cherchent à rendre accessible la formation artistique à des adolescents - des démarches qui tendent à les toucher dans leurs aspirations - je vais m'inspirer dans cet article du contexte où je travaille, celui de l'Ontario français, et en donner un bref aperçu avant de préciser le cadre conceptuel et méthodologique de la présente recherche. Par la suite, j'expliciterai 
la manière dont la formation artistique reçue à l'école et l'expérience de stage peuvent s'avérer complémentaires à l'aide d'une étude de cas.

\section{Contexte de recherche}

2 L'Ontario, province située au centre du Canada et à côté du Québec, compte environ 582855 francophones parmi une population totale de 12029895 de personnes, soit 4,8 \% de la population répartie sur une superficie qui dépasse celles de la France et l'Espagne combinées (Office des affaires francophones de l'Ontario, 2009). Malgré cette situation, les Francophones de cette province forment un réseau bien branché, comme un village virtuel, car ils ont dû au cours de leur histoire lutter pour faire reconnaître leurs droits. Il en résulte une prise de conscience de l'indissociabilité des liens famille, école et communauté, car toutes ces instances sociales sont mises à contribution dans l'appui nécessaire à la construction identitaire des élèves fréquentant les écoles de langue française. C'est pourquoi, les programmes spécialisés en arts offerts à l'école revêtent tant d'importance pour les Franco - Ontariens. D'une part, ils agissent comme gage de survie de la culture. D'autre part, ils constituent un lieu de formation de la relève artistique de l'affirmation d'une identité francophone qui pourrait être fantomatique dans l'ensemble de ce continent nord-américain.

De plus, comme un grand nombre d'adolescents vivent une insertion sur le marché du travail dès 16 ans, la mise en œuvre de programmes spéciaux incluant des stages en milieux professionnels permet d'adapter la formation à des besoins spécifiques plus près de la réalité de jeunes déjà habitués à exercer un métier à temps partiel. Le programme Majeure Haute Spécialisation en Arts et Culture, dont il est question dans cet article, a été conçu en ce sens. Élaboré en 2006, ce programme est réservé à l'élève âgé de 16 à 18 ans qui complète les deux dernières années de scolarité de ses études au secondaire. Il tend à favoriser la transition entre l'école secondaire et les options offertes dans les collèges et universités ainsi que sur le marché du travail. Il inclut des stages en milieux professionnels, ce qui incite à une prise de conscience de la diversité des métiers reliés au monde des arts et donne l'occasion de prendre une expérience reconnue tant par les institutions culturelles que scolaires puisque l'élève qui s'y inscrit reçoit une mention spéciale sur son diplôme d'études du secondaire, ce qui contribue à lui faciliter l'accès à des programmes spécialisés en arts au niveau postsecondaire.

\section{Cadre conceptuel et méthodologique}

3 Dans l'étude de la construction identitaire, le concept d'identité est le premier que j'interpelle dans cet article parce qu'il est, au c ?ur du questionnement sous-jacent à ce passé, présent et perspectives d'avenir qu'expriment les participants lorsqu'ils parlent de leurs expériences et de leurs représentations d'eux-mêmes. Pour aborder ce concept, je me réfère, d'une part, à MUCCHIELLI (1986) ainsi qu'aux concepts de soi et d'expérience (eGINGRAS, 2000 ; DEWEY, 2005; SEVIGNY, 2003) et, d'autre part, à la perspective systémique de la créativité (CSIKSZENTMIHALYI, 1999; 2001 ; 2006 ; CSIKSZENTMIHALYI et Schneider $2000 ; 2001$ ). L'amalgame de ces assises conceptuelles me permet d'amorcer une réflexion au sujet de l'importance de la reconnaissance de la créativité dans un contexte artistique et d'enrichir le regard porté sur la formation artistique et la construction identitaire chez des adolescents, par exemple, sur la manière dont le 
sentiment de confiance concourt à l'affirmation d'un potentiel artistique et aux sentiments de valeur, d'autonomie, d'appartenance, voire d'existence.

En ce qui a trait au cadre méthodologique, je privilégie une approche de nature qualitative/interprétative (SAVOIE-ZAJC, 2000) et la principale stratégie de collecte de données qui a permis d'entrer en contact avec neuf élèves âgés de 16,17 et 18 ans est l'entrevue individuelle. Il ne s'agit aucunement de comparaison entre participants, chaque témoignage constitue une étude de cas qui donne un aperçu de la manière dont il est possible d'adapter un programme d'études dans lequel l'inclusion de stages en milieu artistique professionnel incite une réflexion tant chez l'élève, le milieu éducationnel que dans la communauté. Afin de montrer la complémentarité de la formation reçue à l'école et l'expérience vécue lors d'un stage en milieu artistique professionnel, les trois parties suivantes se centrent plus particulièrement sur le cas de Marcel, élève inscrit en arts visuels et médiatiques.

\section{La formation artistique reçue à l'école}

4 Marcel est en 7 e année, il n'a que 12 ans, lorsqu'il a l'idée de devenir réalisateur au cinéma. Il regarde fréquemment des films et trouve les génériques vraiment intéressants. Cela lui permet de constater la complexité du métier et l'exigence de travailler en équipe. Plus il y réfléchit, plus il se voit réalisateur, même si ce choix de carrière lui semble $a$ priori peu accessible.

À cette époque, Marcel étudie dans un contexte où il a la possibilité de s'inscrire à un programme spécialisé en arts soit en art dramatique, en arts visuels ou en musique. Pour faciliter le choix de s'inscrire ou non à un tel programme, Marcel suit d'abord, à l'âge de 12 et 13 ans, une formation où il explore son potentiel dans chacune de ces trois disciplines artistiques. Au cours de cette formation, Marcel a également la possibilité de discuter de ses aptitudes et de son choix avec ses pairs, ses parents, ses enseignants, l'orienteur de l'école et les responsables du programme.

Cette formation et ces échanges incitent Marcel à s'inscrire au programme spécialisé en arts visuels parce qu'une partie de ce programme touche les arts médiatiques. Ce programme lui offre alors la possibilité de suivre tout au cours de ses études au secondaire un cours de soixante-quinze minutes par jour pendant quatre ans consécutifs en arts visuels. Il comprend diverses expériences de création réalisées sous la supervision d'enseignants spécialistes ou sous celle d'artistes invités qui viennent partager leurs expertises. Il comporte également un ensemble d'expériences esthétiques lors de sorties éducatives ou sur le site même de l'école dans un local réservé à la réalisation d'expositions. Il lui donne l'occasion de se doter de référents culturels et de se servir de logiciels informatiques qui lui permettent de mieux comprendre les exigences techniques de la réalisation de films. Puis à 16 ans, lorsque le coordonnateur des programmes spécialisés en arts parle de la spécificité d'un autre programme, Majeure Haute Spécialisation en Arts et Culture, Marcel décide de s'y engager aussi parce qu'il veut faire des stages dans un milieu de production cinématographique. Il ne tient cependant pas à poursuivre une formation uniquement en arts et comme il le mentionne lui-même dans le témoignage qui suit, il décide de suivre une diversité de cours pour avoir des options variées lorsque viendra le temps de faire un choix de programme au postsecondaire :

"Quand je me suis inscrit au programme de Majeure, je ne voulais pas me fermer des portes et rester juste en arts. J'ai donc pris toutes mes sciences, puis mes maths.

C'est pour ça que ça ne me dérange pas de faire le stage l'été parce que comme ça je 
peux prendre tous les cours dont j'ai besoin. Après ma 12e année, je veux aller à l'université en Études cinématographiques. J'aimerais aller dans une université où je peux aussi continuer à faire du sport, faire partie d'une équipe de volley-ball. J'aimerais vraiment faire les deux : le cinéma et le sport. »

5 Pour Marcel, avoir des intérêts diversifiés contribue à ses chances de saisir des réalités différentes et de les intégrer à des éléments de fictions dans des scénarios. Selon lui, ces choix ne vont pas à l'encontre de sa passion pour le cinéma, mais au contraire l'aident à avoir des idées originales et à explorer des univers qui peuvent faire partie du quotidien de personnages des films qu'il aimerait concevoir. Tout en reconnaissant l'importance de la formation artistique reçue à l'école, Marcel insiste dans son témoignage sur l'importance de la complémentarité de cette formation avec les apprentissages réalisés en stages.

\section{L'expérience de l'art en stage}

6 À l'été de ses 17 ans, Marcel amorce son stage et se rend travailler pendant quatre semaines consécutives dans une compagnie qui loue de l'équipement vidéo et où il $\mathrm{y} a$ aussi une salle de montage. Comme c'est l'été, il y a moins d'activités programmées et il y a des jours où Marcel a moins de tâches à accomplir. Loin de rester désoeuvré, Marcel en profite pour écrire un scénario et élabore un projet de court métrage. Les contacts qu'il développe avec les gens de la boîte incitent ces derniers à lui faire confiance et à lui donner accès à l'équipement disponible pour réaliser le court métrage qu'il vient d'imaginer.

« Dans mon stage, j'ai pris connaissance de tout l'équipement vidéographique. Il y a beaucoup de choses sur l'équipement que je ne connaissais pas, sur certains éclairages, les câbles, les plans. J'ai pu jouer avec l'équipement. C'est aussi moi qui recevais tout l'équipement en salle vidéo. Je suis allé filmer avec un producteur. Ça m'a permis d'apprendre comment se déroule une journée de tournage et comment il faut s'organiser pour passer d'une place à l'autre. Le producteur m'a montré quoi faire sur un plateau, comment diriger les acteurs. Après, j'ai eu la chance de faire mon propre film qui dure environ quinze minutes. J'ai écrit mon propre scénario, j'ai fait des auditions, trouvé mes acteurs. Ils ont accepté de me prêter une caméra et tout l'équipement pendant toute une journée. J'ai filmé moi-même. J'ai appris à bien diriger une équipe. Par la suite, j’ai fait tout le montage, créé ma propre musique.»

Cette expérience s'avère marquante pour plusieurs raisons. D'une part, même si Marcel connaît les différentes étapes de réalisation d'un court métrage, être en action et le produire lui-même l'oblige à prendre conscience de la complexité des relations humaines mises en cause. D'autre part, en devenant réalisateur d'un court métrage, Marcel va audelà de la conception et passe à la réalisation d'un projet, il voit comment ses personnages sont incarnés, quel investissement de temps la réalisation d'un film exige et surtout quel niveau d'engagement est nécessaire de la part de chaque membre de l'équipe. Il réalise également que c'est une partie de son monde imaginaire qui prend forme et devient sujet d'échanges entre les personnes qui collaborent avec lui. Il prend réellement plaisir à ce travail et vit une expérience inoubliable de nature optimale comme l'entend CSIKSZENTMIHALYI (2006). La période du montage constitue aussi un moment important où il approfondit sa réflexion sur la signification de l'histoire qu'il met en scène et sur la manière dont il interagit avec des pairs lors de la production. Puis, lorsque le film est présenté, il peut en discuter avec les artistes qui travaillent à ce 
moment-là à l'entreprise où il fait son stage. Cet échange de commentaires avec des professionnels constitue un autre moment marquant de son stage. Marcel admire le travail de ces artistes. Même s'ils ne sont pas des célébrités reconnues internationalement, il est au courant de leur cheminement professionnel et, grâce à cette expérience et à ces échanges, il comprend tous les efforts nécessaires pour bâtir une carrière de réalisateur. Par le dialogue établi, il évalue également la qualité de son film et de son expérience tout en constatant ce qu'il serait souhaitable d'améliorer lors de la prochaine expérience de production cinématographique.

Lorsqu'ils traitent de l'alternance travail - études, HARDY et MENARD (2008) soulignent l'importance de l'accompagnement des enseignants et des tuteurs afin d'offrir un encadrement propice aux apprentissages. Comme le montre l'exemple du contexte où Marcel réalise son stage, la flexibilité de l'encadrement d'un stage peut donner libre cours à une créativité dans une production artistique qui rend tangible les apprentissages réalisés. Les besoins d'accompagnement ne sont pas tous les mêmes d'une personne à une autre (CIFALI, BOURASSA et THEBERGE, sous presse ; VIAL, M. et CAPARROS-MENCACCI, 2007) et Marcel en arrive à bénéficier de la latitude de son lieu de stage. Il ne faut cependant pas tenir pour acquis qu'il en serait de même pour tous les adolescents placés dans une telle situation.

\section{La complémentarité des apprentissages à l'école et en stage}

8 L'expérience dont Marcel profite en stage continue par la suite de se répercuter dans la formation artistique reçue dans le contexte scolaire. À l'automne, dans le cadre d'un cours d'arts médiatiques, Marcel doit produire d'autres courts métrages, agir en collaboration avec des membres d'équipes, visionner les différentes productions réalisées par ses pairs, en discuter et présenter la réflexion sous-jacente à ses films. L'enseignant qui supervise alors le travail du groupe d'élèves prend rapidement conscience de l'expertise que Marcel a acquise lors de son expérience estivale et l'encourage à approfondir ses connaissances techniques et conceptuelles de la réalisation cinématographique. Après quelques semaines, il lui demande de présenter l'un des courts métrages réalisés dans le cadre du cours à un festival de films qui regroupe des productions régionales du milieu artistique.

Dans la suite des événements vécus en stage et de la formation reçue à l'école, cette expérience inscrit une autre marque dans le parcours de Marcel. Elle rend encore plus tangible l'importance d'établir des relations avec une communauté de pratique. Cette production devient une occasion additionnelle d'établir un dialogue avec le milieu artistique professionnel présent lors du festival. Encore une fois, ce qui a été appris en contexte scolaire se reflète à l'extérieur de l'école, dans un milieu artistique. Marcel intègre ainsi un processus d'identification à une profession en en apprivoisant la culture et les rouages. Son parcours témoigne aussi de l'engagement d'un ensemble de personnes qui l'appuient. Par exemple, les enseignants en arts visuels de l'école que fréquente Marcel reconnaissent les aptitudes de cet élève et l'encouragent à poursuivre des études dans le domaine artistique. Le coordonnateur du programme de Majeure Haute Spécialisation en Arts et Culture s'entretient personnellement à plusieurs reprises avec Marcel et ses parents. L'orienteur de l'école établit aussi des contacts personnalisés avec Marcel et favorise dans ses interventions une diversité dans le choix de matières 
d'apprentissage, ce qui convient aux goûts de cet élève.

Dans le même ordre d'idées, les parents de Marcel constatent que la formation que reçoit leur enfant lui permet de s'exprimer et d'évoluer.

«Au début, mes parents étaient d'accord pour que je m'inscrive au programme de

la Majeure, mais pas plus que ça. Après, quand j'ai fait mon stage, ils m'ont dit: «Peut-être que ça va t'aider dans le futur pour entrer à l'université, que ça va t'aider à savoir si tu aimes vraiment ça et à avoir beaucoup d'expérience. »

Maintenant, je sens que mes deux parents m'encouragent dans tout ce que je fais ».

Il est heureux pour Marcel et son entourage que cela se soit déroulé de cette manière. Par contre, on peut imaginer une situation inverse où le milieu éducationnel et celui de la communauté de pratique reconnaissent peu la valeur de ce qui est créé. Qu'adviendrait-il alors du rêve artistique de l'élève? En art, le rapport à soi et le rapport d'altérité s'inscrivent dans une continuité dans un rapport d'affirmation de soi dans un environnement : ce qui est produit vient de soi et est une partie de soi. L'artiste est une personne qui affirme son unicité et sa créativité sous diverses formes. Lorsque celles-ci sont reconnues, elles deviennent des référents culturels pour la communauté ainsi que dans d'autres contextes sociaux (THEBERGE, 2007, 2009). Cependant, un manque de reconnaissance de la production artistique peut provoquer une rupture dans la manière de se représenter soi-même et initier une remise en question fondamentale. Pour un adolescent qui rêve de faire de l'art, prendre conscience que ce rêve ne se réalisera peutêtre pas peut susciter un choc et c'est pourquoi l'accompagnement s'avère d'une importance capitale dans la mise en oeuvre de stage en milieu professionnel, entre autres, pour éviter que l'élève vive une trop grande solitude lorsque son idéal ne correspond pas à ce qui se passe en réalité.

\section{CONCLUSION}

Comme l'exemple de l'expérience de Marcel permet de le montrer, les deux programmes de formation artistique - programme spécialisé en arts et Majeure Haute Spécialisation en Arts et Culture, instaurés par le ministère de l'Éducation de l'Ontario, peuvent favoriser la richesse de l'expérience d'apprentissage en art. Si ces programmes et ces stages sont mis en pratique de manière à donner un appui tangible à des êtres en évolution, à leur donner la chance de cerner et de vivre une passion ou comme le dit CSIKSZENTMIHALYI (2006) une expérience optimale, l'apport du milieu éducationnel se bonifie lorsqu'il est conjugué à celui du milieu artistique. Un ensemble de liens se tissent entre les artistes et les jeunes qui rêvent de faire de l'art, entre la culture et les membres d'une communauté qui affirment une entité par les arts. Cependant, il importe de réaliser que formation artistique et la signification qu'on lui accorde comptent autant dans le rapport à soi, le rapport d'altérité et celui à l'environnement que la manière dont sont mis en œuvre des programmes de stage comme celui de la Majeure Haute Spécialisation en Arts et Culture dont il question dans cet article. En soi, l'expérience de Marcel est riche de signification, car elle peut contribuer non seulement à faire ressortir les apports de collaborations entre institutions scolaires, collégiales et universitaires, mais aussi inciter un dialogue sur les besoins de formation en art des adolescents. Reste à suivre l'évolution de telles initiatives afin de documenter et de comprendre la manière dont elles peuvent prendre ancrage dans une culture organisationnelle et être bénéfiques tant pour la construction identitaire de l'élève que pour sa formation artistique. 


\section{BIBLIOGRAPHIE}

ASSOCIATION CANADIENNE D'ÉDUCATION DE LANGUE FRANÇAISE (2006). Cadre d'orientation en construction identitaire. Québec : Association canadienne d'éducation de langue française.

BUJOLD, C. et GINGRAS. M. (2000). Choix professionnel et développement de carrière. Théories et recherches . 2e édition. Boucherville (Québec) : Gaëtan Morin éditeur.

CIFALI, M., BOURASSA M. et THÉBERgE, M. (Dir.) (sous PRESSE). Accompagner. Une éthique clinique. Paris : L'Harmattan.

CSIKSZENTMIHALYI, M. (1999). Implications of a Systems Perspective for the study of Creativity. In R. J. Sternberg (Éd.). Handbook of Creativity (pp. 313-335). Cambridge, Massachusetts: Cambridge University Press.

CSIKSZENTMIHALYI, M. (2001). Talented teenagers: The roots of success and failure. Cambridge, Massachusetts: Cambridge University Press.

CSIKSZENTMihalyi, M. (2006). La créativité : Psychologie de la découverte et de l'invention. Paris: Éditions Robert Laffont.

CSIKSZENTMIHALYI, M. et SCHNEIDER, B. (2000). Becoming adult: How teenagers prepare for the world of work. New York, New York: Basic Books.

CSIKSZENTMIHALYI, M. et SCHNEIDER, B. (2001). Conditions for optimal development in adolescence: An expérimential approach. Lawrence Erlbaum Assoc.

DEWEY, J. (2005), L'art comme expérience, œuvres philosophiques III, Traduit sous la direction de jean-Pierre Cometti. Publications de l'Université de Pau, Éditions Farrago.

FÉDÉRATION CULTURELLE CANADIENNE FRANÇAISE (2009). La trousse du passeur culturel. Ottawa : Fédération culturelle canadienne française.

HARDY, M. et MÉNARD, L. (2008). Alternance travail-études : les effets des stages dans la formation professionnelle des élèves. Revue des sciences de l'éducation. XXXIV(3). 689-709.

MINISTÈRE DE L'ÉDUCATION DE L'ONTARIO. (2004). Politique d'aménagement linguistique. Toronto, Ontario : Imprimeur de la Reine pour l'Ontario.

MINISTÈRE DE L'ÉDUCATION DE L'ONTARIO. (2006). Majeure Haute spécialisation. Consulté le 18 août 2009. http ://www.edu.gov.on.ca/fre/studentsuccess/pathways/shsm/shsm_fact_sheet.pdf

Mucchielli, A. (1986). L'identité. Collection Que sais-je ? Paris, France : Presses universitaires de France.

OFFICE DES AFFAIRES FRANCOPHONES DE L'ONTARIO. Recensement de 2006. Consulté le 18 août 2009. http ://www.ofa.gov.on.ca/fr/franco-carte.html

SAVOIE-ZAJC, L. (2000). La recherche qualitative / interprétative en éducation. In T.

Karsenti et L. Savoie-Zajc Introduction à la recherche en éducation (pp. 171-198). Sherbrooke : Éditions du CRP.

SÉVIGNY, R. (2003). Expérience. In J. Barus-Michel, E. Enriquez, et A. Lévy (Éds.), Vocabulaire de Psychosociologie : Références et positions, (pp. 128-133). Ramonville Saint-Agne, France : Éditions érès. 
THÉBERGE, M. (2007) L'école comme lieu d'ancrage de la culture. Québec : Revue de recherche en éducation musicale (26), 25-38. http ://www.mus.ulaval.ca/reem/REM26_Theberge05.pdf

THÉBERGE, M. (2009). L'art suscite l'art. Entr'Acte : Revue de réflexion sur le théâtre francoontarien. Ottawa : Théâtre Action.

VIAL, M. et CAPARROS-MENCACCI, N. (2007). L'accompagnement professionnel ? Méthode à l'usage des praticiens exerçant une fonction éducative. Bruxelles, Belgique : De Boeck.

\section{RÉSUMÉS}

Dans les cinq dernières années, plusieurs approches d'éducation artistique et politiques linguistiques ont été mises en oeuvre dans le contexte de la francophonie canadienne pour favoriser la construction identitaire de l'élève. Par exemple, des programmes spécialisés en arts ont été mis sur pied afin de favoriser une concertation entre les contextes de formation scolaire, collégiale, universitaire et en cours d'emploi. Le programme Majeure Haute Spécialisation en Arts et Culture conçu par le ministère de l'éducation de l'Ontario répond à cette visée et l'étude de cas, dont il est question dans cet article, témoigne de la manière dont peut se vivre une insertion sur le marché du travail dès 16 ans, dans le cadre d'un stage réalisé en arts médiatiques en milieu professionnel.

In the last five years, several linguistic policies and approaches to art education have been implemented in the Canadian francophone context, in order to promote the building of students' sense of identity. For example, specialized programs in the arts have been established to encourage a consensus within the training contexts at school, at college, at university, and on the job. Created by the Ontario Ministry of Education, the Specialist High Skills Major in Arts and Culture Program corresponds to this goal, and the case study presented in this article shows how one can experience the integration into the work force at age 16, within a media arts practicum in a professional environment.

INDEX

Mots-clés : adolescence, construction identitaire, éducation artistique, minorité linguistique

\section{AUTEUR}

\section{MARIETTE THÉBERGE}

Université d'Ottawa 